\title{
An Osgood Type Regularity Criterion for the 3D Boussinesq Equations
}

\author{
Qiang Wu, ${ }^{1,2}$ Lin $\mathrm{Hu}^{2}$ and Guili Liu ${ }^{3}$ \\ ${ }^{1}$ School of Resources and Safety Engineering, Central South University, Changsha, Hunan 410075, China \\ 2 Jiangxi University of Science and Technology, Ganzhou, Jiangxi 341000, China \\ ${ }^{3}$ Department of Basic Teaching, Harbin Finance University, Harbin 150030, Heilongjiang, China
}

Correspondence should be addressed to Guili Liu; guili2005_liu@126.com

Received 2 November 2013; Accepted 2 February 2014; Published 11 March 2014

Academic Editors: D. Baleanu and H. Jafari

Copyright (C) 2014 Qiang Wu et al. This is an open access article distributed under the Creative Commons Attribution License, which permits unrestricted use, distribution, and reproduction in any medium, provided the original work is properly cited.

We consider the three-dimensional Boussinesq equations, and obtain an Osgood type regularity criterion in terms of the velocity gradient.

\section{Introduction}

In this paper, we consider the following three-dimensional (3D) Boussinesq equations with the incompressibility condition:

$$
\begin{gathered}
\mathbf{u}_{t}+(\mathbf{u} \cdot \nabla) \mathbf{u}-\Delta \mathbf{u}+\nabla \pi=\theta \mathbf{e}_{3}, \\
\theta_{t}+(\mathbf{u} \cdot \nabla) \theta-\Delta \theta=0, \\
\nabla \cdot \mathbf{u}=0, \\
\mathbf{u}(x, 0)=\mathbf{u}_{0}, \quad \theta(x, 0)=\theta_{0},
\end{gathered}
$$

where $\mathbf{u}=\left(u_{1}(x, t), u_{2}(x, t), u_{3}(x, t)\right)$ is the fluid velocity, $\pi=\pi(x, t)$ is a scalar pressure, and $\theta=\theta(x, t)$ is the scalar temperature, while $\mathbf{u}_{0}$ and $\theta_{0}$ are the prescribed initial velocity and temperature, respectively, with $\nabla \cdot \mathbf{u}_{0}=0$.

In case $\theta=0$, (1) reduces to the incompressible NavierStokes equations. The regularity of its weak solutions and the existence of global strong solutions are important open problems; see [1-3]. Starting with $[4,5]$, there have been a lot of literatures devoted to finding sufficient conditions (which now are called regularity criteria) to ensure the smoothness of the solutions; see [6-16] and so forth. Since the convective terms $(\mathbf{u} \cdot \nabla) \mathbf{u}$ are the same in the Navier-Stokes equations and Boussinesq equations, the authors also consider the regularity conditions for (1). In particular, Qiu et al. [17] obtained Serrin type regularity condition:

$$
\mathbf{u} \in L^{p}\left(0, T ; L^{q}\left(\mathbb{R}^{3}\right)\right), \quad \frac{2}{p}+\frac{3}{q}=1, \quad 3<q \leqslant \infty .
$$

The extension to the multiplier spaces was established by the same authors in [18]. For the Besov-type regularity criterion, Fan and Zhou [19] and Ishimura and Morimoto [20] showed the following regularity conditions:

$$
\begin{gathered}
\nabla \times \mathbf{u} \in L^{1}\left(0, T ; \dot{B}_{\infty, \infty}^{0}\left(\mathbb{R}^{3}\right)\right), \\
\nabla \mathbf{u} \in L^{1}\left(0, T ; L^{\infty}\left(\mathbb{R}^{3}\right)\right) .
\end{gathered}
$$

Zhang $[21,22]$ then considers the regularity criterion in terms of the pressure or its gradient. The readers are also referred to [23] for generalized models.

Motivated by [24-26], we will improve (3) as in the following.

Theorem 1. Let $\left(\mathbf{u}_{0}, \theta_{0}\right) \in H^{1}\left(\mathbb{R}^{3}\right)$. Assume that $(\mathbf{u}, \theta)$ is the smooth solution to (1) with the initial data $\left(\mathbf{u}_{0}, \theta_{0}\right)$ for $0 \leqslant t<$ T. If

$$
\sup _{2 \leqslant q<\infty} \int_{0}^{T} \frac{\left\|\bar{S}_{q} \nabla \mathbf{u}\right\|_{L^{\infty}}}{q \ln q}<\infty,
$$


then the solution $(\mathbf{u}, \theta)$ can be extended after time $t=T$. Here, $\dot{\Delta}_{k}$ denotes the Fourier localization operator and $\Delta S_{q}=$ $\sum_{l=-q}^{q} \dot{\Delta}_{l}$

Remark 2. The Osgood type condition (4) is weaker than (3). Notice that, for $q \in[2, \infty)$, we have

$$
\frac{\left\|\bar{S}_{q} \nabla \mathbf{u}\right\|_{L^{\infty}}}{q \ln q} \leqslant \frac{1}{q \ln q} \sum_{l=-q}^{q}\left\|\dot{\Delta}_{l}(\nabla \times \mathbf{u})\right\|_{L^{\infty}} \leqslant C\|\nabla \times \mathbf{u}\|_{\dot{B}_{\infty, \infty}^{0}} .
$$

The rest of this paper is organized as follows. In Section 2, we recall the definition of Besov spaces and some interpolation inequalities. Section 3 is devoted to proving Theorem 1.

\section{Preliminaries}

Let $\delta\left(\mathbb{R}^{3}\right)$ be the Schwartz class of rapidly decreasing functions. For $f \in \delta\left(\mathbb{R}^{3}\right)$, its Fourier transform $\mathscr{F} f=\widehat{f}$ is defined by

$$
\widehat{f}(\xi)=\int_{\mathbb{R}^{3}} f(x) e^{-i x \cdot \xi} d x .
$$

Let us choose a nonnegative radial function $\varphi \in \mathcal{S}\left(\mathbb{R}^{3}\right)$ such that

$$
0 \leqslant \widehat{\varphi}(\xi) \leqslant 1, \quad \widehat{\varphi}(\xi)= \begin{cases}1, & \text { if }|\xi| \leqslant 1, \\ 0, & \text { if }|\xi| \geqslant 2,\end{cases}
$$

and let

$$
\begin{gathered}
\psi(x)=\varphi(x)-2^{-3} \varphi\left(\frac{x}{2}\right), \\
\varphi_{j}(x)=2^{3 j} \varphi\left(2^{j} x\right), \quad \psi_{j}(x)=2^{3 j} \psi\left(2^{j} x\right), \quad j \in \mathbb{Z} .
\end{gathered}
$$

For $j \in \mathbb{Z}$, the Littlewood-Paley projection operators $S_{j}$ and $\dot{\Delta}_{j}$ are, respectively, defined by

$$
S_{j} f=\varphi_{j} * f, \quad \dot{\Delta}_{j} f=\psi_{j} * f .
$$

Observe that $\dot{\Delta}_{j}=S_{j}-S_{j-1}$. Also, it is easy to check that if $f \in L^{2}\left(\mathbb{R}^{3}\right)$, then

$$
S_{j} f \longrightarrow 0, \quad \text { as } j \longrightarrow-\infty ; \quad S_{j} f \longrightarrow f, \quad \text { as } j \longrightarrow+\infty \text {, }
$$

in the $L^{2}$ sense. By telescoping the series, we thus have the following Littlewood-Paley decomposition:

$$
f=\sum_{j=-\infty}^{+\infty} \dot{\Delta}_{j} f
$$

for all $f \in L^{2}\left(\mathbb{R}^{3}\right)$, where the summation is the $L^{2}$ sense. Notice that

$$
\dot{\Delta}_{j} f=\sum_{l=j-2}^{j+2} \dot{\Delta}_{l} \dot{\Delta}_{j} f=\sum_{l=j-2}^{j+2} \psi_{l} * \psi_{j} * f ;
$$

then from Young's inequality, it readily follows that

$$
\left\|\dot{\Delta}_{j} f\right\|_{L^{q}} \leqslant C 2^{3 j(1 / p-1 / q)}\left\|\dot{\Delta}_{j} f\right\|_{L^{p}},
$$

where $1 \leqslant p \leqslant q \leqslant \infty$ and $C$ is an absolute constant independent of $f$ and $j$.

Let $-\infty<s<\infty, 1 \leqslant p, q \leqslant \infty$; the homogeneous Besov space $\dot{B}_{p, q}^{s}$ is defined by the full-dyadic decomposition such that

$$
\dot{B}_{p, q}^{s}=\left\{f \in \mathscr{Z}^{\prime}\left(\mathbb{R}^{3}\right) ;\|f\|_{\dot{B}_{p, q}^{s}}<\infty\right\}
$$

where

$$
\|f\|_{\dot{B}_{p, q}^{s}}=\left\|\left\{2^{j s}\left\|\dot{\Delta}_{j} f\right\|_{L^{p}}\right\}_{j=-\infty}^{+\infty}\right\|_{\ell^{q}}
$$

and $\mathscr{Z}^{\prime}\left(\mathbb{R}^{3}\right)$ is the dual space of

$$
\mathscr{Z}\left(\mathbb{R}^{3}\right)=\left\{f \in \mathcal{S}\left(\mathbb{R}^{3}\right) ; D^{\alpha} \widehat{f}(0)=0, \forall \alpha \in \mathbb{N}^{3}\right\} .
$$

Also, it is well known that

$$
\dot{H}^{s}\left(\mathbb{R}^{3}\right)=\dot{B}_{2,2}^{s}\left(\mathbb{R}^{3}\right), \quad \forall s \in \mathbb{R} .
$$

We refer to [27] for more detailed properties.

\section{Proof of Theorem 1}

This section is devoted to proving Theorem 1. From standard continuity arguments, we need to only provide the uniform $H^{1}$ bounds of the solution $(\mathbf{u}, \theta)$.

Taking the inner products of $(1)_{1}$ with $-\Delta \mathbf{u},(1)_{2}$ with $-\Delta \theta$, we obtain by adding together that

$$
\begin{aligned}
& \frac{1}{2} \frac{d}{d t}\|\nabla(\mathbf{u}, \theta)\|_{L^{2}}^{2}+\|\Delta(\mathbf{u}, \theta)\|_{L^{2}}^{2} \\
& =\int_{\mathbb{R}^{3}}[(\mathbf{u} \cdot \nabla) \mathbf{u}] \cdot \Delta \mathbf{u} d x-\int_{\mathbb{R}^{3}} \theta \Delta u_{3} \mathrm{~d} x \\
& \quad+\int_{\mathbb{R}^{3}}[(\mathbf{u} \cdot \nabla) \theta] \cdot \Delta \theta \mathrm{d} x \\
& =\int_{\mathbb{R}^{3}} \partial_{k} \theta \partial_{k} u_{3} \mathrm{~d} x \\
& \quad-\int_{\mathbb{R}^{3}} \partial_{k} u_{j}\left(\partial_{j} u_{i} \partial_{k} u_{i}+\partial_{j} \theta \partial_{k} \theta\right) \mathrm{d} x \\
& \equiv I+J .
\end{aligned}
$$

For $I$, we use Hölder's inequality to get

$$
I_{1} \leqslant \frac{1}{2}\|\nabla(\mathbf{u}, \theta)\|_{L^{2}}^{2} .
$$

For $J$, applying the Littilewood-Paley decomposition as in (11), we get

$$
\nabla \mathbf{u}=\sum_{l<-q} \dot{\Delta} \nabla \mathbf{u}+\sum_{l=-q}^{q} \dot{\Delta} \nabla \mathbf{u}+\sum_{l>q} \dot{\Delta} \nabla \mathbf{u},
$$


where $q$ is positive integral to be determined later on. Plugging (20) into $J$, we see that

$$
\begin{aligned}
J \leqslant & \sum_{l<-q} \int_{\mathbb{R}^{3}}\left|\dot{\Delta}_{l} \nabla \mathbf{u}\right| \cdot|\nabla(\mathbf{u}, \theta)|^{2} \mathrm{~d} x \\
& +\int_{\mathbb{R}^{3}}\left|\sum_{l=-q}^{q} \dot{\Delta}_{l} \nabla \mathbf{u}\right| \cdot|\nabla(\mathbf{u}, \theta)|^{2} \mathrm{~d} x \\
& +\sum_{l>q} \int_{\mathbb{R}^{3}}\left|\dot{\Delta}_{l} \nabla \mathbf{u}\right| \cdot|\nabla(\mathbf{u}, \theta)|^{2} \mathrm{~d} x \\
\equiv & J_{1}+J_{2}+J_{3} .
\end{aligned}
$$

For $J_{1}$, we dominate as

$$
\begin{aligned}
J_{1} & \leqslant \sum_{l<-q}\left\|\dot{\Delta}_{l} \nabla \mathbf{u}\right\|_{L^{\infty}}\|\nabla(\mathbf{u}, \theta)\|_{L^{2}}^{2} \\
& \leqslant C \sum_{l<-q} 2^{3 l / 2}\left\|\dot{\Delta}_{l} \nabla \mathbf{u}\right\|_{L^{2}}\|\nabla(\mathbf{u}, \theta)\|_{L^{2}}^{2} \quad(\text { by }(13)) \\
& \leqslant C\left(\sum_{l<-q} 2^{(3 l / 2) \cdot 2}\right)^{1 / 2} \cdot\left(\sum_{l<-q}\left\|\dot{\Delta}_{l} \nabla \mathbf{u}\right\|_{L^{2}}^{2}\right)^{1 / 2}\|\nabla(\mathbf{u}, \theta)\|_{L^{2}}^{2} \\
& \leqslant C 2^{-3 q / 2}\|\nabla \mathbf{u}\|_{L^{2}}\|\nabla(\mathbf{u}, \theta)\|_{L^{2}}^{2} \quad(\text { by }(17)) \\
& =\left[C 2^{-q / 2}\|\nabla(\mathbf{u}, \theta)\|_{L^{2}}\right]^{3} .
\end{aligned}
$$

For $J_{2}$, we have

$$
\begin{aligned}
J_{2} & =\int_{\mathbb{R}^{3}}\left|\bar{S}_{q} \nabla \mathbf{u}\right| \cdot|\nabla(\mathbf{u}, \theta)|^{2} \mathrm{~d} x \\
& \leqslant\left\|\bar{S}_{q} \nabla \mathbf{u}\right\|_{L^{\infty}}\|\nabla(\mathbf{u}, \theta)\|_{L^{2}}^{2} .
\end{aligned}
$$

Finally, for $J_{3}$, we estimate as

$$
\begin{aligned}
J_{3} & \leqslant \sum_{l>q}\left\|\Delta_{l} \nabla \mathbf{u}\right\|_{L^{3}}\|\nabla(\mathbf{u}, \theta)\|_{L^{3}}^{2} \\
& \leqslant C \sum_{l>q} 2^{l / 2}\left\|\Delta_{l} \nabla \mathbf{u}\right\|_{L^{2}}\|\nabla(\mathbf{u}, \theta)\|_{L^{2}}\|\Delta(\mathbf{u}, \theta)\|_{L^{2}}
\end{aligned}
$$

(by (13) and Gagliardo-Nireberg inequality)

$$
\begin{aligned}
\leqslant & C\left(\sum_{l>q} 2^{-(l / 2) \cdot 2}\right)^{1 / 2} \cdot\left(\sum_{l>q} 2^{l \cdot 2}\left\|\dot{\Delta}_{l} \nabla \mathbf{u}\right\|_{L^{2}}^{2}\right)^{1 / 2} \\
& \times\|\nabla(\mathbf{u}, \theta)\|_{L^{2}}\|\Delta(\mathbf{u}, \theta)\|_{L^{2}} \\
\leqslant & {\left[C 2^{-q / 2}\|\nabla(\mathbf{u}, \theta)\|_{L^{2}}\right]\|\Delta(\mathbf{u}, \theta)\|_{L^{2}}^{2} \quad \text { (by (17)). } }
\end{aligned}
$$

Gathering (22), (23), and (24) together and plugging them into (21), we deduce

$$
\begin{aligned}
J \leqslant & {\left[C 2^{-q / 2}\|\nabla(\mathbf{u}, \theta)\|_{L^{2}}\right]^{3} } \\
& +\left\|\bar{S}_{q} \nabla \mathbf{u}\right\|_{L^{\infty}}\|\nabla(\mathbf{u}, \theta)\|_{L^{2}}^{2} \\
& +\left[C 2^{-q / 2}\|\nabla(\mathbf{u}, \theta)\|_{L^{2}}\right]\|\Delta(\mathbf{u}, \theta)\|_{L^{2}}^{2} .
\end{aligned}
$$

Substituting (19) and (25) into (18), we find

$$
\begin{aligned}
\frac{1}{2} \frac{d}{d t}\|\nabla(\mathbf{u}, \theta)\|_{L^{2}}^{2}+\|\Delta(\mathbf{u}, \theta)\|_{L^{2}}^{2} \\
\leqslant \frac{1}{2}\|\nabla(\mathbf{u}, \theta)\|_{L^{2}}^{2}+\left[C 2^{-q / 2}\|\nabla(\mathbf{u}, \theta)\|_{L^{2}}\right]^{3} \\
+\frac{\left\|\bar{S}_{q} \nabla \mathbf{u}\right\|_{L^{\infty}}}{q \ln q} \cdot q \ln q\|\nabla(\mathbf{u}, \theta)\|_{L^{2}}^{2} \\
+\left[C 2^{-q / 2}\|\nabla(\mathbf{u}, \theta)\|_{L^{2}}\right]\|\Delta(\mathbf{u}, \theta)\|_{L^{2}}^{2} .
\end{aligned}
$$

Taking

$$
q=\left[\frac{2}{\ln 2 \ln ^{+}\left(C\|\nabla(\mathbf{u}, \theta)\|_{L^{2}}\right)}\right]+1,
$$

where $[t]$ is the largest integer smaller than $t \in \mathbb{R}$ and $\ln ^{+} t=$ $\ln (e+t)$, then (26) implies that

$$
\begin{aligned}
& \frac{d}{d t}\|\nabla(\mathbf{u}, \theta)\|_{L^{2}}^{2} \\
& \leqslant\|\nabla(\mathbf{u}, \theta)\|_{L^{2}}^{2}+C \\
& \quad+\frac{\left\|\bar{S}_{q} \nabla \mathbf{u}\right\|_{L^{\infty}}}{q \ln q} \ln ^{+}\left(\|\nabla(\mathbf{u}, \theta)\|_{L^{2}}\right) \ln ^{+} \ln ^{+}\left(\|\nabla(\mathbf{u}, \theta)\|_{L^{2}}\right) \\
& \quad \times\|\nabla(\mathbf{u}, \theta)\|_{L^{2}}^{2} .
\end{aligned}
$$

Applying Gronwall inequality three times, we deduce

$$
\begin{aligned}
& \|\nabla(\mathbf{u}, \theta)\|_{L^{2}}^{2}+\int_{0}^{t}\|\Delta(\mathbf{u}, \theta)\|_{L^{2}} \mathrm{~d} \tau \\
& \quad \leqslant C \exp \exp \exp \left(\int_{0}^{t} \frac{\left\|\bar{S}_{q} \nabla \mathbf{u}\right\|_{L^{\infty}}}{q \ln q} \mathrm{~d} \tau\right) .
\end{aligned}
$$

Recalling (4), we see the solution $(\mathbf{u}, \theta)$ is uniformly bounded in $L^{\infty}\left(0, T ; H^{1}\left(\mathbb{R}^{3}\right)\right)$. This completes the proof of Theorem 1 .

\section{Conflict of Interests}

The authors declare that there is no conflict of interests regarding the publication of this paper. 


\section{Acknowledgments}

This work was supported by the NSF of China (no. 11326238, no. 11326138, and no. 11101101), the Science Foundation of Jiangxi Provincial Department of Education (no. GJJ13374 and no. GJJ13658), and the Youth Natural Science Foundation of Jiangxi Province (20132BAB211007).

\section{References}

[1] E. Hopf, "Über die Anfangswertaufgabe für die hydrodynamischen Grundgleichungen," Mathematische Nachrichten, vol. 4, pp. 213-231, 1951.

[2] P. G. Lemarié-Rieusset, Recent Developments in the NavierStokes Problem, vol. 431, Chapman \& Hall/CRC, Boca Raton, Fla, USA, 2002.

[3] J. Leray, "Sur le mouvement d'un liquide visqueux emplissant l'espace," Acta Mathematica, vol. 63, no. 1, pp. 193-248, 1934.

[4] J. Serrin, "On the interior regularity of weak solutions of the Navier-Stokes equations," Archive for Rational Mechanics and Analysis, vol. 9, no. 1, pp. 187-195, 1962.

[5] J. Serrin, "The initial value problem for the Navier-Stokes equations," in Nonlinear Problems, R. E. Langer, Ed., pp. 69-98, University of Wisconsin Press, Madison, Wis, USA, 1963.

[6] X. W. He and S. Gala, "Regularity criterion for weak solutions to the Navier-Stokes equations in terms of the pressure in the class $L^{2}\left(0, T ; B_{\infty, \infty}^{-1}\left(\mathbb{R}^{3}\right)\right)$," Nonlinear Analysis, Real World Applications, vol. 12, no. 6, pp. 3602-3607, 2011.

[7] J. Neustupa, A. Novotný, and P. Penel, "An interior regularity of a weak solution to the Navier-Stokes equations in dependence on one component of velocity," in Topics in Mathematical Fluid Mechanics, vol. 10 of Quaderni di Matematica, pp. 163-183, Seconda Università degli Studi di Napoli, Caserta, Italy, 2002.

[8] Z. J. Zhang, "A Serrin-type regularity criterion for the NavierStokes equations via one velocity component," Communications on Pure and Applied Analysis, vol. 12, no. 1, pp. 117-124, 2013.

[9] Z. J. Zhang, "A remark on the regularity criterion for the $3 \mathrm{D}$ Navier-Stokes equations involving the gradient of one velocity component," Journal of Mathematical Analysis and Applications, vol. 414, no. 1, pp. 472-479, 2014.

[10] Z. J. Zhang, Z.-A. Yao, P. Li, C. C. Guo, and M. Lu, "Two new regularity criteria for the $3 \mathrm{D}$ Navier-Stokes equations via two entries of the velocity gradient," Acta Applicandae Mathematicae, vol. 123, no. 1, pp. 43-52, 2013.

[11] Z. J. Zhang, D. X. Zhong, and L. Hu, "A new regularity criterion for the 3D Navier-Stokes equations via two entries of the velocity gradient tensor," Acta Applicandae Mathematicae, vol. 129, no. 1, pp. 175-181, 2014.

[12] Z. J. Zhang, Z. A. Yao, M. Lu, and L. D. Ni, "Some Serrintype regularity criteria for weak solutions to the Navier-Stokes equations," Journal of Mathematical Physics, vol. 52, no. 5, Article ID 053103, 2011.

[13] Z. J. Zhang, P. Li, and D. X. Zhong, "Navier-Stokes equations with regularity in two entries of the velocity gradient tensor," Applied Mathematics and Computation, vol. 228, pp. 546-551, 2014.

[14] Y. Zhou, "A new regularity criterion for weak solutions to the Navier-Stokes equations," Journal des Mathematiques Pures et Appliquees, vol. 84, no. 11, pp. 1496-1514, 2005.

[15] Y. Zhou and M. Pokorný, "On a regularity criterion for the Navier-Stokes equations involving gradient of one velocity component," Journal of Mathematical Physics, vol. 50, no. 12, Article ID 123514, 11 pages, 2009.

[16] Y. Zhou and M. Pokorný, "On the regularity of the solutions of the Navier-Stokes equations via one velocity component," Nonlinearity, vol. 23, no. 5, pp. 1097-1107, 2010.

[17] H. Qiu, Y. Du, and Z. A. Yao, "Serrin-type blow-up criteria for 3D Boussinesq equations," Applicable Analysis, vol. 89, no. 10, pp. 1603-1613, 2010.

[18] H. Qiu, Y. Du, and Z. Yao, "Blow-up criteria for 3D Boussinesq equations in the multiplier space," Communications in Nonlinear Science and Numerical Simulation, vol. 16, no. 4, pp. 18201824, 2011.

[19] J. S. Fan and Y. Zhou, "A note on regularity criterion for the 3D Boussinesq system with partial viscosity," Applied Mathematics Letters, vol. 22, no. 5, pp. 802-805, 2009.

[20] N. Ishimura and H. Morimoto, "Remarks on the blow-up criterion for the 3-D Boussinesq equations," Mathematical Models and Methods in Applied Sciences, vol. 9, no. 9, pp. 1323$1332,1999$.

[21] Z. J. Zhang, "A remark on the regularity criterion for the 3D Boussinesq equations via the pressure gradient," Abstract and Applied Analysis, vol. 2014, Article ID 510924, 4 pages, 2014.

[22] Z. J. Zhang, "A logarithmically improved regularity criterion for the $3 \mathrm{D}$ Boussinesq equations via the pressure," Acta Aplicandae Mathematicae, 2013.

[23] B. Mehdinejadiani, H. Jafari, and D. Baleanu, "Derivation of a fractional Boussinesq equation for modelling uncon-fined groundwater," The European Physical Journal Special Topics, vol. 222, no. 8, pp. 1805-1812, 2013.

[24] Q. Zhang, "Refined blow-up criterion for the 3D magnetohydrodynamics equations," Applicable Analysis, vol. 92, no. 12, pp. 2590-2599, 2013.

[25] Z. J. Zhang and S. Gala, "Osgood type regularity criterion for the 3D Newton-Boussinesq equation," Electronic Journal of Differential Equations, vol. 2013, no. 223, pp. 1-6, 2013.

[26] Z. J. Zhang, T. Tang, and L. H. Liu, "An Osgood type regularity criterion for the liquid crystal flows," Nonlinear Differerntial Equations and Applications, 2013.

[27] H. Triebel, Interpolation Theory, Function Spaces, Differential Operators, vol. 18, North-Holland, Amsterdam, The Netherlands, 1978. 


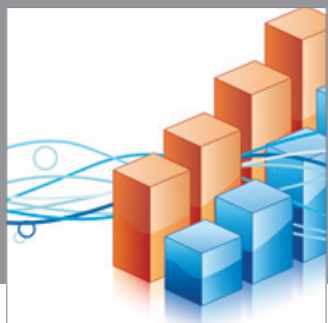

Advances in

Operations Research

mansans

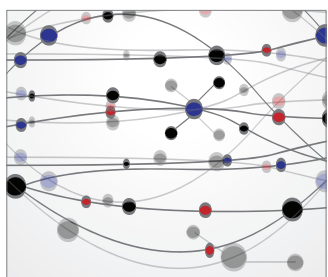

The Scientific World Journal
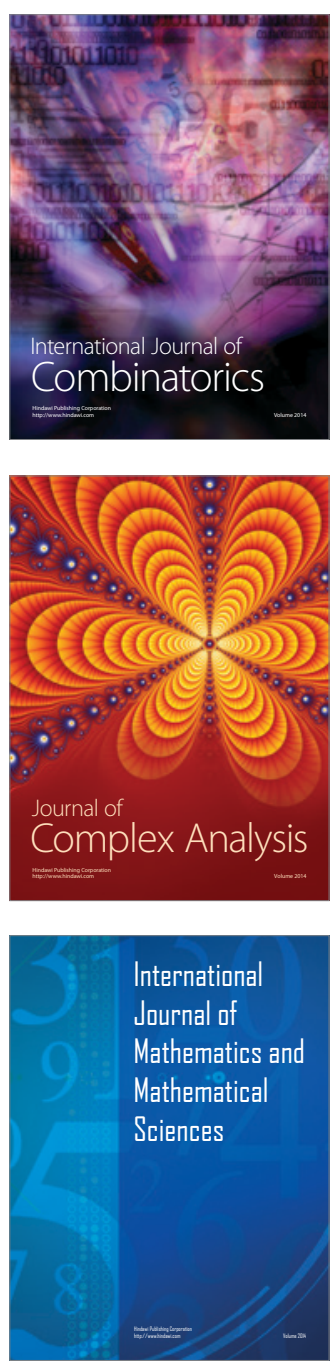
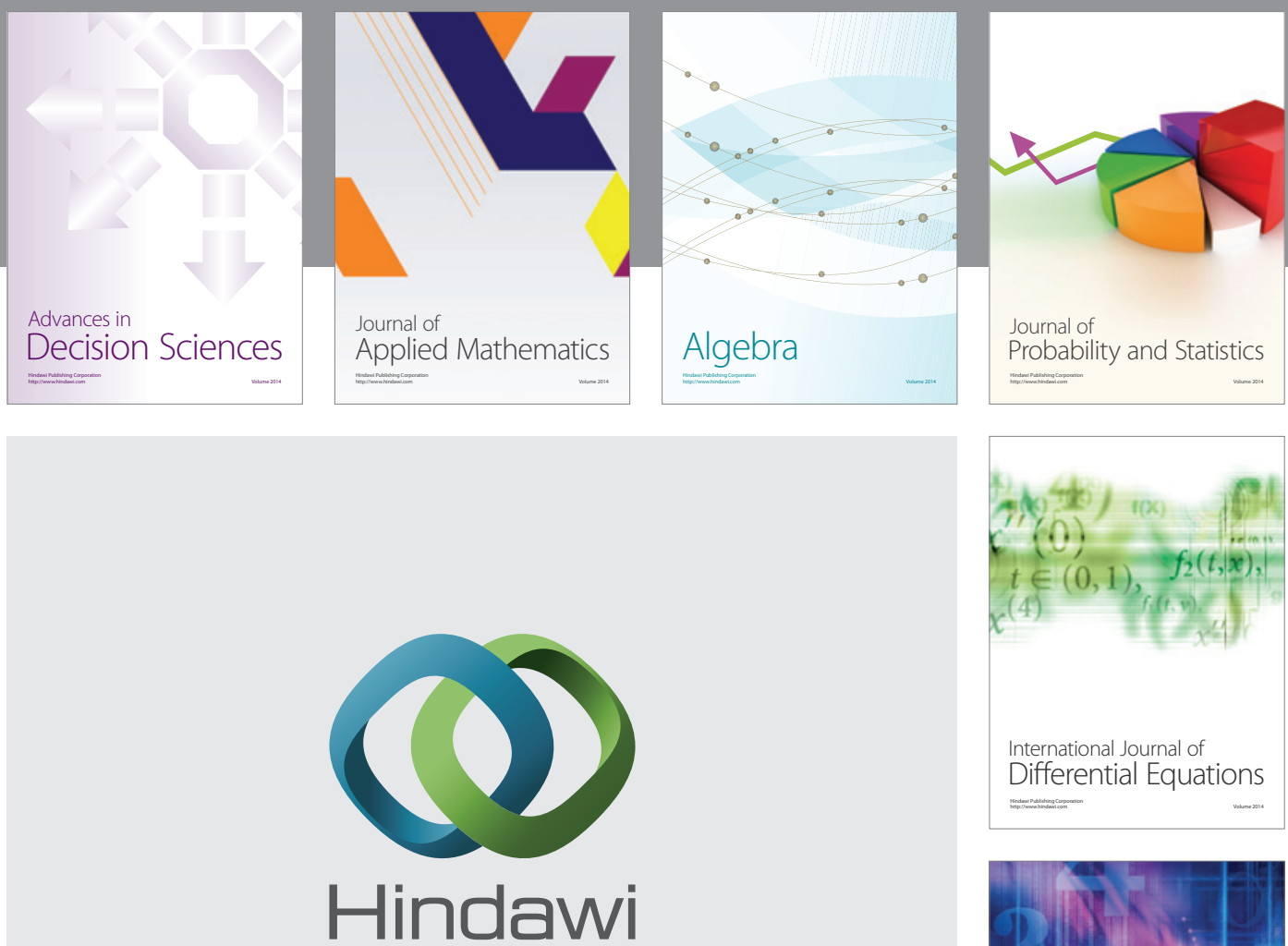

Submit your manuscripts at http://www.hindawi.com
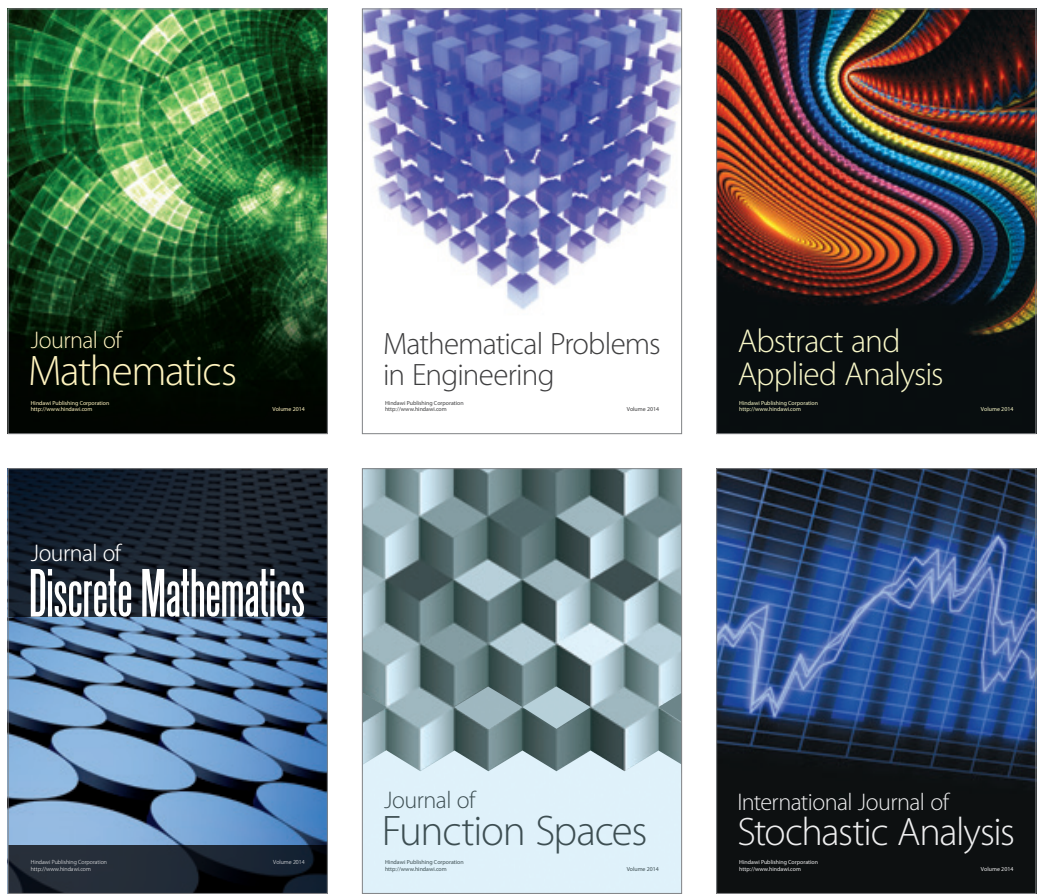

Journal of

Function Spaces

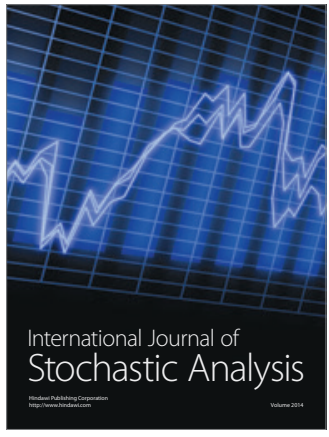

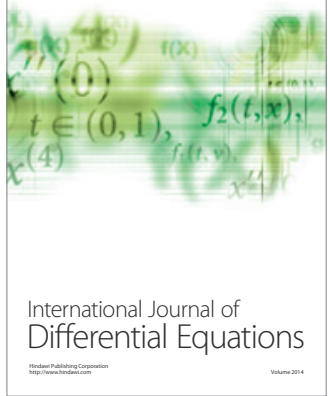
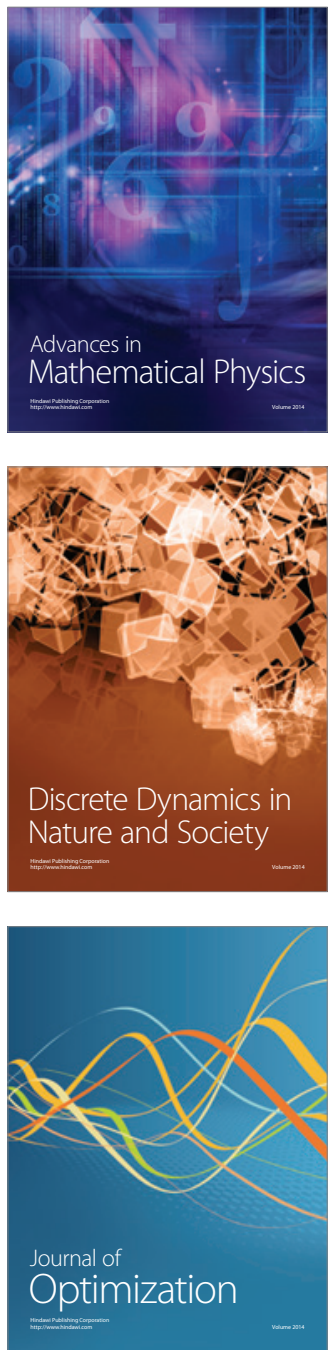